Dhaka Univ. J. Biol. Sci. 21(1): 47-53, 2012 (January)

\title{
THE IMPORTANCE OF PRE-HATCHING PATERNAL CARE ON OFFSPRING FITNESS IN BURYING BEETLE, NICROPHORUS VESPILLOIDES HERBST.
}

\author{
SHARMIN MUSA* \\ Faculty of Life Sciences, University of Manchester, Stopford Building, \\ Oxford Road, Manchester, M13 9PT, UK
}

Key words: Parental care, Offspring fitness, Burying beetles, Nicrophorus vespilloides

\begin{abstract}
A male-removal experiment was performed to determine if the value of male parental care depended on the timing of the help. In the experiment male parent was removed before the carcass was prepared for breeding, after partial carcass preparation, after complete carcass preparation, before direct care for the larvae and males were allowed to disperse naturally after caring for the larvae. It was found that where the male provided complete pre-hatching care or both preand post-hatching care offspring were larger and in better condition. Mass of offspring at eclosion was affected by male removal $\left(\mathrm{F}_{3,1266}=5.087, \mathrm{p}=0.002\right)$ though size of offspring was not affected by the treatment group. Complete prehatching care had a positive effect on development compared to limited care $\left(\mathrm{F}_{3,1267}=8.501, \mathrm{p}<0.000\right)$ but this effect disappeared if males remained after the larvae hatched. Larval survivorship did not vary among treatments $\left(\mathrm{F}_{3,122}=0.531\right.$, $\mathrm{p}=0.662)$.
\end{abstract}

\section{Introduction}

Biparental care is uncommon in invertebrates ${ }^{(1,2,3)}$. In burying beetles both parents provide care for the larvae both before and after larval hatching. They provision the offspring directly by regurgitating pre-digested carrion into the mouthparts of the larvae. Females stay with the brood until larval development is complete and when they disperse from the carcass, but males disperse several days earlier ${ }^{(4-7)}$.

Single males and females are equally competent as parents, but under biparental conditions males perform less direct care of the offspring ${ }^{(8-10)}$. Males can benefit by remaining with the carcass by gaining access to a known resource ${ }^{(11)}$, defending the carcass and the brood from intra- and interspecific intruders ${ }^{(6,11-13)}$. There may be some benefit to the female in terms of future reproductive success ${ }^{(14)}$. However, there is no evidence of a beneficial effect of male assistance in direct care for the larvae on offspring components of fitness beyond uniparental female care ${ }^{(10,14,)}$. A previously neglected question for burying beetles is the importance of timing of male assistance in parental care. Studies on birds have shown that the timing of male removal can be important $t^{(17,18)}$.

\footnotetext{
*Present address: Department of Zoology, University of Dhaka, Dhaka-1000, Bangladesh. <sharminsumi@yahoo.com>
} 
In burying beetles, pre-hatching care, which includes the preparation of the carcass for breeding, is important for offspring survival (19). However, this is also the time period when male parents do most of their foraging. Thus, the costs of pre-hatching care (in terms of resources lost to the offspring) differ from the costs to the offspring of the presence of parents during post-hatching care. Benefits of the presence of the parents may also vary depending on the timing, but this has not been examined. In this study a male-removal experiment in Nicrophorus vespilloides Herbst designed to test for different benefits of male participation in care at different time points. In the most extreme treatment male parent was removed immediately after mating so that the female received no assistance in care. In a second treatment, males remained with the female long enough to provide assistance in the burial and some of the preparation of the carcass. In a third treatment, males took full part in carcass preparation prior to larval hatching, but provided no care directly to the larvae. In the last group male were allowed to desert naturally and therefore took full part in both pre- and post- hatching parental care.

\section{Materials and Methods}

The beetles used in this study were from an outbred laboratory population derived from over 100 wild-caught Nicrophorus vespilloides females trapped in a deciduous forest in August 2003 at Sunbank Wood, Manchester, England. Beetles were housed individually from the day they dispersed from the carcass in clear plastic containers $(17 \times$ $12 \mathrm{~cm}$ area and $6 \mathrm{~cm}$ high) under a $16: 8 \mathrm{hrs}$ light:dark cycle at $20 \pm 2^{\circ} \mathrm{C}$, and fed decapitated mealworms (Tenebrio) twice a week.

One hundred and twenty pairs of sexually mature virgin males and females were randomly selected from the laboratory stock population. Each pair was kept in plastic box for $24 \mathrm{hrs}(17 \times 12 \times 6 \mathrm{~cm})$ filled with soil during which time they were allowed to mate. Pairs were randomly assigned to each of four treatment groups, which differed with respect to duration of male care: (1) No male care. In this treatment, males were removed $24 \mathrm{hrs}$ after placing them with a female and before providing a previously frozen mouse carcass $(n=30),(2)$ Limited pre-hatching care. Males were removed 24 hours after providing a mouse, allowing limited pre-hatching care $(\mathrm{n}=30)$, (3) Full prehatching care (no post-hatching care). Males were removed the day when the larvae were first seen in the crypt made by the parents, which is approximately 3 - 4 days after introducing the carcass $(\mathrm{n}=32)$ and (4) Full pre- and post-hatching care (control treatment). Males were removed the day they deserted naturally $(n=34)$. Males that were not observed in the near vicinity of carcass (i.e., within $1 \mathrm{~cm}$ of the carcass) for two consecutive days were scored as deserted. Previously frozen mice ranged in size from 9.5 to $17.5 \mathrm{~g}$ were used as carcass. 
Number of larvae that dispersed from the carcass for each family, mass of each larvae at dispersal, mass at adult eclosion and size of adult offspring was recorded as offspring fitness. Mass was determined by weighing each larva to the nearest $0.01 \mathrm{mg}$ using an Ohaus analytical balance. Size was determined from digital images of the offspring reaching the adult stage using NIH ImageJ (http:// rsb.info.nih.gov/nih-image/) by measuring the length of the pronotum along the suture line in the middle of the pronotum ${ }^{(20)}$.

Two clearly defined stages were measured to investigate the effects of treatments on development: (i) Duration of larval development on the carcass and (ii) duration of the "wandering stage", larval development off the carcass when the larvae no longer forage. Wandering is measured from dispersal to the beginning of pupation(21). All larvae disperse together from the carcass when the resource is exhausted.

Different ANOVA models were used to analyse if removal of male parent had any effect on offspring fitness. Nested ANOVA with family nested under treatment for variables that are influenced by genetic differences and therefore more similar within families than between families ${ }^{(22)}$. ANCOVA (Analysis of Co-variance) were used to measure traits that did not vary among family members, such as the time of dispersal of larvae from the carcass and the number of larvae at dispersal. In these analyses the number of degrees of freedom equalled the number of families. Carcass size was included as a covariate and data were transformed when not normally distributed.

\section{Results and Discussions}

There was no significant effect of the timing of male removal on the number of offspring that dispersed (Fig. $1 ; \mathrm{F}_{3,121}=2.103, \mathrm{p}=0.103$ ). In addition, duration of larval development on the carcass was not affected by the timing of male removal $\left(\mathrm{F}_{3,120}=1.021\right.$, $p=0.386$ ). Carcass size did not have a significant effect on either trait.

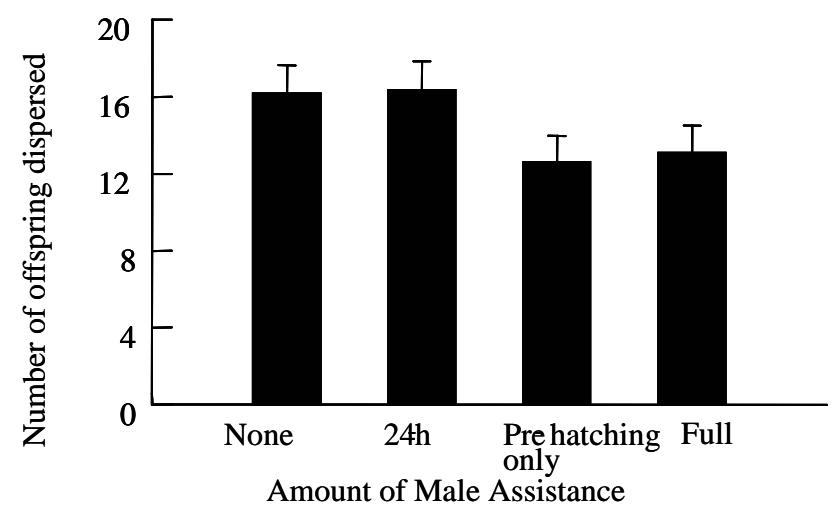

Fig. 1. The effect of pre- and post-hatching care on the number of offspring that disperse from the carcass in each family (Mean \pm 1 SE). 
As expected, there was a significant family effect on mass at eclosion $\left(\mathrm{F}_{105,1266}=8.652\right.$, $p<0.001$ ). Controlling for this family effect, the timing of male removal also had a significant effect on the mass of each larva at eclosion (Fig. 2a; $F_{3,1266}=5.087, p=0.002$ ). The offspring of families where the male had access to the carcass until larval hatching and in the control groups were heavier as adults compared to the offspring of families of the other treatment groups.

Adult size was significantly influenced by family membership $\left(\mathrm{F}_{105,1180}=11.198, \mathrm{p}<\right.$ 0.001). Controlling for this effect, the size of offspring that emerged to adulthood was not affected by the timing of male removal (Fig. $2 b ; F_{3,1180}=1.101, p=0.347$ ). However, there was a trend for broods that received no male parental care to be smaller than broods that received at least some male parental care. There was a significant correlation between adult weight and size (Pearson's $r=0.759, \mathrm{n}=1289, \mathrm{p}<0.001$ ).

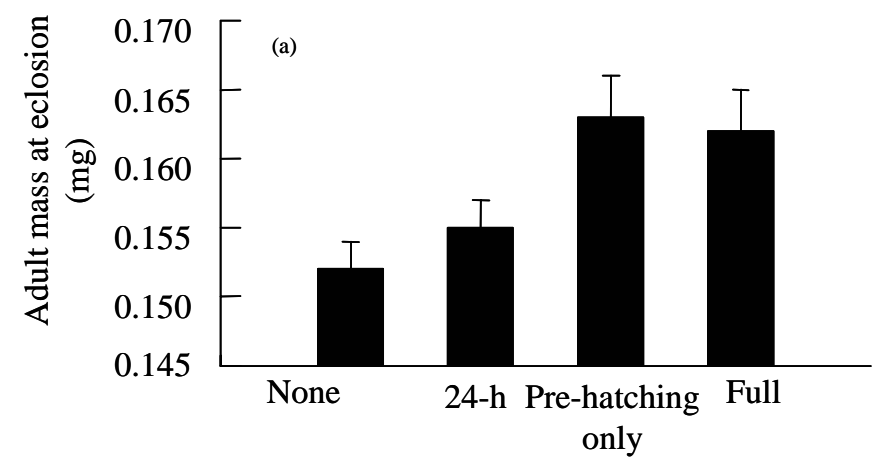

Amount of male assistance

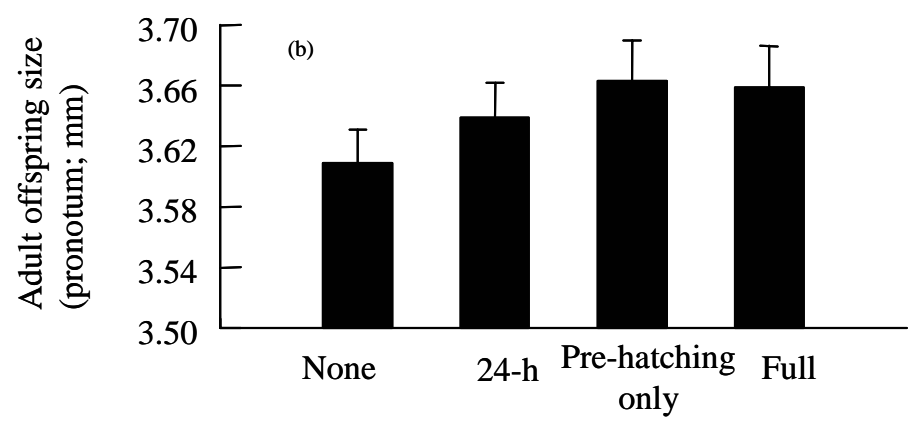

Amount of male assistance

Fig. 2. The effect of pre-and post-hatching care on (a) mass and (b) size of offspring.

Consistent with previous research showing genetic influences on development, family membership influenced the proportion of time spent in the wandering stage $\left(\mathrm{F}_{105,1180}=6.723, \mathrm{p}<0.001\right)$. The timing of male removal also had a significant influence on the proportion of time spent in the wandering stage (Fig. 3, $F_{3,1267}=8.501, p<0.000$ ). This 
was due to a single treatment shortening the proportion of time spent wandering. Offspring from families where the male was removed at larval hatching had shorter wandering stages than offspring from families of the other treatments. Offspring survival varied from 66 to $73 \%$, and was not significantly different among the different treatments $\left(\mathrm{F}_{3,122}=0.531, \mathrm{p}=0.662\right)$.

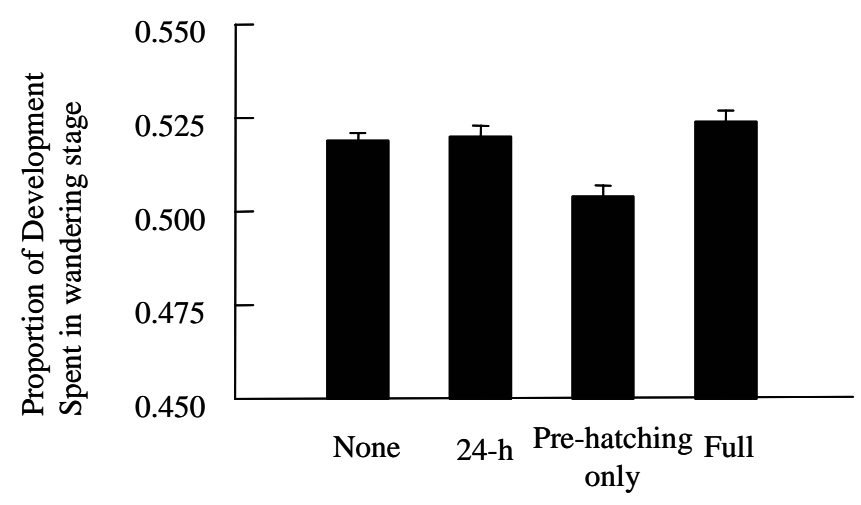

Amount of male assistance

Fig 3. The effect of pre- and post-hatching care on offspring development.

Eggert et al. (1998) ${ }^{(19)}$ performed a similar experiment where they examined the critical period of uniparental female care, and also found that increasing amounts of prehatching care increased larval body mass. It therefore seems likely that pre-hatching male care compensated for the time that females were away from the carcass laying eggs - a period of time that can last several hours and occur repeatedly over a day ${ }^{(23)}$.

Male assistance during carcass preparation and direct care of the larvae, therefore, has a positive effect on the offspring. However, broods that only received pre-hatching care and broods that received full pre- and post-hatching care did not differ. This suggests that male care is important before larval hatching when the carcass is prepared for breeding. Male assistance in preparing the carcass for breeding may only be important when single females are time constrained because they must allocate time between egg-laying and carcass preparation.

The value of the assistance of the male in provisioning the larvae after they hatch remains unexplained. In the present study larval development off the carcass suggests that the presence of males on the carcass after the larvae arrive may even have a subtle negative effect on offspring fitness. Larvae that received pre-hatching biparental care but that did not share the carcass with the male after hatching spent less time in the wandering stage, the stage where larvae appear to be most vulnerable(24). This study suggest that males should abandon their families at the time of larval hatching. However, as pointed out by Scott ${ }^{(11,25)}$, Scott and Gladstein ${ }^{(26)}$ and Trumbo ${ }^{(12,6)}$, male defence against 
conspecific competitors may be a very important component of burying beetle breeding systems. If so, there may be trade-offs between the benefits of assistance in defence and costs of resource competition that select for male persistence ${ }^{(26)}$. However, the involvement of males in post-hatching provisioning of larvae remains unexplained in burying beetles. This assistance in direct care of the larvae may incur no benefits when the female is present, but simply reflect the maintenance of behaviour through genetic correlations from selection on females. Alternatively or in addition, males may be selected to retain the propensity to provide direct care for the offspring in case the female dies or deserts the broods. Thus, the possibility cannot be excluded that the expression of male provisioning under biparental conditions simply reflects a non-adaptive response to conditions and stimuli and is not beneficial to the offspring.

\section{Acknowledgements}

This is a part of Ph.D. thesis of the author (SM). The author is grateful to Professor Allen J. Moore and Dr. Per T. Smiseth, University of Manchester for their guidance and support. Author is also grateful to Ministry of Science and Technology, Government of the People's Republic of Bangladesh for financial support.

\section{References}

1. Wilson EO 1971. The Insect Societies. Harvard University Press, Cambridge, MA, pp. 562.

2. Clutton-Brock TH 1991. The Evolution of Parental Care. Princeton University Press, Princeton, New Jersey, pp. 368.

3. Choe JC. and BJ Crespi 1997. The evolution of Social Behavior in Insects and Arachnids: Cambridge University Press, Cambridge, pp. 552.

4. Barlett J 1988. Male mating success and paternal care in Nicrophorus vespilloides (Coleoptera: Silphidae). Behav. Ecol. Sociobiol. 23: 297-303.

5. Scott MP and JFA Traniello 1990. Behavioural and ecological correlates of male and female parental and reproductive success in the burying beetle Nicrophorus orbicollis. Anim. Behav. 39: 274-283.

6. Trumbo ST 1991. Reproductive benefits and the duration of paternal care in a biparental burying beetle, Necrophorus orbicollis. Behaviour 117: 82-105.

7. Schwarz HH and JK Müller 1992. The dispersal behaviour of the phoretic mite Poecilochirus carabi (Mesostigmata, Parasitidae): Adaptation to the breeding biology of its carrier Necrophorus vespilloides (Coleoptera, Silphidae). Oecologia 89: 487-493.

8. Fetherston IA, MP Scott and JFA Traniello 1990. Parental care in burying beetles: the organization of male and female brood care behaviour. Ethology 85: 177-190.

9. Rauter CM and AJ Moore 2004. Time constraints and trade-offs among parental care behaviours: effects of brood size, sex, and loss of mate. Anim. Behav. 68: 695-702.

10. Smiseth PT, C Dawson, E Varley and AJ Moore 2005. How do caring males respond to mate loss? Differential response by males and females. Anim. Behav. 69: 551-559. 
11. Scott MP 1990. Brood guarding and the evolution of male parental care in burying beetles. Behav. Ecol. Sociobiol. 26: 31-39.

12. Trumbo ST 1990. Interference competition among burying beetles (Silphidae: Nicrophorus). Ecol. Ento. 15: 347-355.

13. Robertson IC 1993. Nest intrusions, infanticide, and parental care in the burying beetle, Nicrophorus orbicollis (Coleoptera: Silphidae). J. Zool. 231: 583-593.

14. Jenkins EV, C Morris and S Blackman 2000. Delayed benefits of paternal care in the burying beetle Nicrophorus vespilloides. Anim. Behav. 60: 443-451.

15. Scott MP 1989. Male parental care and reproductive success in the burying beetle Nicrophorus orbicollis. Journal. Insect. Behav. 2: 133-137.

16. Reinking M and JK Müller 1990. The benefit of parental care in the burying beetle, Necrophorus orbicollis (Coleoptera: Silphidae). J. Zool (London) 231: 583-593.

17. Trumbo ST and AG Fernandez 1995. Regulation of brood size by male parents and cues employed to assess resource size by burying beetles. Etho. Eco. Evo. 7: 313-322.

18. Müller JK, A-K Eggert and SK Sakaluk, SK 1998. Carcass maintenance and biparental brood care in burying beetles: are males redundant? Ecol. Ento. 23: 195-200.

19. Satou A, T Nisimura and H Numata 2001. Cost and necessity of parental care in the burying beetle Nicrophorus quadripunctatus. Zool. Science. 18: 975-979.

20. Lyon B. and RD Montgomerie 1985. Incubation feeding in snow buntings: female manipulation or indirect male parental care? Behav. Ecol. Sociobiol. 17: 279-284.

21. Sasvári L 1986. Reproductive effort of widowed birds. J. An. Eco. 55: 553-564.

22. Eggert A.-K, M Reinking and JK Müller 1998. Parental care improves offspring survival and growth in burying beetles. Anim. Behav. 55: 97-107.

23. Beeler AE, CM Rauter and AJ Moore 2002. Mate discrimination by females in the burying beetle Nicrophorus orbicollis: the influence of male size on attractiveness to females. Eco. Ento. 17: 1-6.

24. Eggert AK and JK Müller 1997. Biparental care and social evolution in burying beetles: lessons from the larder. In: The Evolution of Social Behavior in Insects and Arachnids. (Choe JC and BJ Crespi Eds.). pp. 216-236. Cambridge University Press, Cambridge, U.K.

25. Rauter CM and AJ Moore 2002. Quantitative genetics of growth and development time in the burying beetle Nicrophorus pustulatus in the presence and absence of post-hatching parental care. Evolution 56: 96-110.

26. Smiseth PT, S Musa and AJ Moore. 2005. Effect of timing of mate loss on parental behaviour of female Nicrophorus vespilloides. Behaviour 143: 293-301.

27. Lock JE, PT Smiseth and AJ Moore 2004. Selection, inheritance and the evolution of parentoffspring interactions. Am. Nat. 164: 1-24.

28. Scott MP 1994. The benefit of paternal assistance in intra- and interspecific competition for the burying beetle Nicrophorus defodiens. Etho. Eco. Evo 6: 537-543.

29. Scott MP and D Gladstein 1993. Calculating males? The duration of paternal care in burying beetles. Evo. Eco. 7: 362-378. 